OAK RIDGE

Y-12

PLANT

LOCKHEED MATTIR

\section{EMERGENCY PLANNING AND COMMUNITY RIGHT-TO-KNOW ACT SECTION 311}

\author{
R. A. EVANS \\ K. J. MARTIN
}

Environmental Compliance Organiration

March 1997

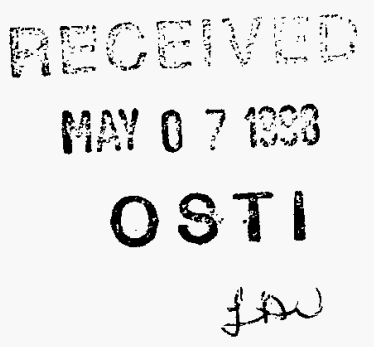

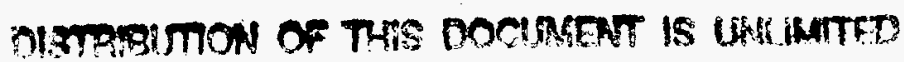

Prepared by the Oak Ridge Y-12 Plant Oak Ridge, Tennessee 37831

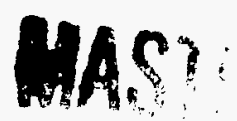
managed by

Lockheed Martin Energy Systems, Inc. for the

U.S. DEPARTMENT OF ENERGY

Under Contract No. DE-AC05-84-OR21400

MANAGED BY

LOCKHEED MARTIN ENERGY SYSTEMS, INC.

FOR THE UNITED STATES

DEPARTMENT OF ENERGY 


\section{LIST OF HAZARDOUS AND EXTREMELY HAZARDOUS CHEMICALS AT THE OAK RIDGE Y-12 PLANT}

The following information reflects changes in the lists of hazardous chemicals present at this facility in amounts equal to or greater than 10,000 pounds and extremely hazardous chemicals present in amounts equal to or greater than 500 pounds or its Threshold Planning Quantity, whichever was less. These lists represent the following:

- List of materials last reported in March 1996 (reference Y/TS-1482),

- Materials to be deleted from list,

- Materials to be added to list, and

- Revised list of materials. 
List of Materials Last Reported

\begin{tabular}{|c|c|c|}
\hline Material & CAS & Hazard Codes ${ }^{\mathrm{a}}$ \\
\hline Acetic Acid & $64-19-7$ & 1,3 \\
\hline Activated Carbon (carbon graphite, synthetic) & $7440-44-0$ & 1,3 \\
\hline Aluminum Chloride (anhydrous) & $7446-70-0$ & 1,5 \\
\hline Argon & $7440-37-1$ & $4^{b}$ \\
\hline Beryllim and Beryllium Compounds & $7440-41-7$ & $1,2,5$ \\
\hline Calcium Choloride & $10043-52-4$ & 1 \\
\hline Calcium Hydroxide & $1305-62-0$ & 1,2 \\
\hline Calcium Metal & $7440-70-2$ & $1,3,5$ \\
\hline Carbon Dioxide (gas) & $124-38-9$ & $4^{b}$ \\
\hline Carbon Dioxide (solid) & $124-38-9$ & 1 \\
\hline Chlorine & $7782-50-5$ & $1,4,5$ \\
\hline Diatomaceous Earth & $61790-53-2$ & 1,2 \\
\hline Diesel (Fuel Oil No. 2) & $-\cdots$ & $1,2,3$ \\
\hline Ferric Sulfate (solution) & $10028-22-5$ & 1 \\
\hline Freon 11 (Trichlorofluormethane) & $75-69-4$ & 1,2 \\
\hline Freon 12 (Dicholrodifluoromethane) & $75-71-8$ & $1,2,4$ \\
\hline Freon 22 (Chlorodifluoromethane) & $75-45-6$ & $1,2,4$ \\
\hline Freon 113 (Trichlorotrifluorethane) & $76-13-1$ & 1,2 \\
\hline Gasoline (unleaded) & ---- & $1,2,3$ \\
\hline
\end{tabular}


List of Materials Last Reported (continued)

\begin{tabular}{|c|c|c|}
\hline Material & $\mathrm{CAS}$ & Hazard Codes ${ }^{\mathrm{a}}$ \\
\hline Helium & $7440-59-7$ & 4 \\
\hline Hydrochloric Acid & $7647-01-0$ & $1,2,4,5$ \\
\hline Hydrogen Fluoride & $7664-39-3$ & $1,2,4$ \\
\hline Isopropyl Alcohol & $67-63-0$ & $1,3,5$ \\
\hline Lithium Chloride & $7447-41-8$ & 1,2 \\
\hline Lithium Deuteride & $13587-16-1$ & $1,2,3,5$ \\
\hline Lithium Hydride & $7580-67-8$ & $1,2,3,5$ \\
\hline Lithium Hydroxide & $1310-65-2$ & $1,2,5$ \\
\hline Magnesium Oxide & $1309-48-4$ & 1,2 \\
\hline Mercury & $7439-97-6$ & 1,2 \\
\hline Methanol (Methyl Alcohol) & $67-56-1$ & $1,2,3$ \\
\hline Nitric Acid & $7697-37-2$ & $1,2,5$ \\
\hline Nitrogen & $7727-37-9$ & 1,4 \\
\hline Oxygen & $7782-44-7$ & 1,4 \\
\hline Petroleum & $\ldots$ & $1,2,3$ \\
\hline Propane & $74-98-6$ & $1,3,4$ \\
\hline Propylene Glycol & $57-55-6$ & 3 \\
\hline
\end{tabular}


List of Materials Last Reported (continued)

\begin{tabular}{|l|l|l|}
\hline \multicolumn{1}{|c|}{ Material } & \multicolumn{1}{|c|}{ CAS } & Hazard Code $^{\mathrm{a}}$ \\
\hline Sodium Bisulfite & $7631-90-5$ & 1 \\
\hline Sodium Chloride & $7647-14-5$ & 1 \\
\hline Sodium Hydroxide & $1310-73-2$ & $1,2,5$ \\
\hline Sodium Hypochlorite & $7681-52-9$ & 1 \\
\hline Sodium Silicofluoride & $16893-85-9$ & 1,5 \\
\hline Sulfuric Acid & $7664-93-9$ & $1,2,5$ \\
\hline \multicolumn{3}{|l}{} \\
\hline Uranium and Uranium Compounds & $7440-61-1$ & $1,2,3$ \\
\hline Urea & $57-13-6$ & 2,3 \\
\hline
\end{tabular}

aHazard Codes:

1. - Immediate (acute) Health Hazard

2. - Delayed (chronic) Health Hazard

3. - Fire Hazard

4. - Sudden Release of Pressure (compressed gases)

5. - Reactive Hazard

'Included also on the basis of its classification as an asphyxiant. 
Materials to be Deleted from List

\begin{tabular}{|l|c|c|}
\hline \multicolumn{1}{|c|}{ Material } & CAS & Hazard Code $^{\mathrm{a}}$ \\
\hline Calcium Metal & $7440-70-2$ & $1,3,5$ \\
\hline \multicolumn{3}{|l|}{} \\
\hline Sodium Silicofluoride & $16893-85-9$ & 1,5 \\
\hline \multicolumn{3}{|l}{} \\
\hline
\end{tabular}

aHazard Codes:

1. - Immediate (acute) Health Hazard

2. - Delayed (chronic) Health Hazard

3. - Fire Hazard

4. - Sudden Release of Pressure (compressed gases)

5. - Reactive Hazard 
Materials to be added to List

\begin{tabular}{|l|c|c|}
\hline \multicolumn{1}{|c|}{ Material } & CAS & Hazard Code $^{\mathrm{a}}$ \\
\hline Sodium Zinc Polyphosphate & $65997-17-3$ & 1,2 \\
\hline \multicolumn{2}{|l}{} \\
\hline
\end{tabular}

aHazard Codes:

1. - Immediate (acute) Health Hazard

2. - Delayed (chronic) Health Hazard

3. - Fire Hazard

4. - Sudden Release of Pressure (compressed gases)

5. - Reactive Hazard

bIncluded also on the basis of its classification as an asphyxiant. 
Revised List of Materials

\begin{tabular}{|c|c|c|}
\hline Material & CAS & Hazard Codes ${ }^{\mathbf{a}}$ \\
\hline Acetic Acid & $64-19-7$ & 1,3 \\
\hline Activated Carbon (carbon graphite, synthetic) & $7440-44-0$ & 1,3 \\
\hline Aluminum Chloride (anhydrous) & $7446-70-0$ & 1,5 \\
\hline Argon & $7440-37-1$ & $4^{b}$ \\
\hline Beryllim and Beryllium Compounds & $7440-41-7$ & $1,2,5$ \\
\hline Calcium Choloride & $10043-52-4$ & 1 \\
\hline Calcium Hydroxide & $1305-62-0$ & 1,2 \\
\hline Carbon Dioxide (gas) & $124-38-9$ & $4^{b}$ \\
\hline Carbon Dioxide (solid) & 124-38-9 & 1 \\
\hline Chlorine & $7782-50-5$ & $1,4,5$ \\
\hline Diatomaceous Earth & $61790-53-2$ & 1,2 \\
\hline Diesel (Fuel Oil No. 2) & $-\cdots$ & $1,2,3$ \\
\hline Ferric Sulfate (solution) & $10028-22-5$ & 1 \\
\hline Freon 11 (Trichlorofluormethane) & $75-69-4$ & 1,2 \\
\hline Freon 12 (Dicholrodifluoromethane) & $75-71-8$ & $1,2,4$ \\
\hline Freon 22 (Chlorodifluoromethane) & $75-45-6$ & $1,2,4$ \\
\hline Freon 113 (Trichlorotrifluorethane) & $76-13-1$ & 1,2 \\
\hline Gasoline (unleaded) & $\ldots$ & $1,2,3$ \\
\hline
\end{tabular}




\section{Revised List of Materials (continued)}

\begin{tabular}{|c|c|c|}
\hline Material & CAS & Hazard Codes ${ }^{\mathrm{a}}$ \\
\hline Helium & $7440-59-7$ & 4 \\
\hline Hydrochloric Acid & $7647-01-0$ & $1,2,4,5$ \\
\hline Hydrogen Fluoride & $7446-39-3$ & $1,2,4$ \\
\hline Isopropyl Alcohol & $67-63-0$ & $1,3,5$ \\
\hline Lithium Chloride & $7447-41-8$ & 1,2 \\
\hline Lithium Deuteride & $13587-16-1$ & $1,2,3,5$ \\
\hline Lithium Hydride & $7580-67-8$ & $1,2,3,5$ \\
\hline Lithium Hydroxide & $1310-65-2$ & $1,2,5$ \\
\hline Magnesium Oxide & $1309-48-4$ & 1,2 \\
\hline Mercury & $7439-97-6$ & 1,2 \\
\hline Methanol (Methyl Alcohol) & $67-56-1$ & $1,2,3$ \\
\hline Nitric Acid & $7697-37-2$ & $1,2,5$ \\
\hline Nitrogen & $7727-37-9$ & 1,4 \\
\hline Oxygen & $7782-44-7$ & 1,4 \\
\hline Petroleum & $\ldots$ & $1,2,3$ \\
\hline Propane & 74-98-6 & $1,3,4$ \\
\hline Propylene Glycol & $57-55-6$ & 3 \\
\hline
\end{tabular}


Revised List of Materials (continued)

\begin{tabular}{|l|l|l|}
\hline \multicolumn{1}{|c|}{ Material } & \multicolumn{1}{c|}{ CAS } & \multicolumn{1}{c|}{ Hazard Codes $^{\mathrm{a}}$} \\
\hline Sodium Bisulfite & $7631-90-5$ & 1 \\
\hline Sodium Chloride & $7647-14-5$ & 1 \\
\hline Sodium Hydroxide & $1310-73-2$ & $1,2,5$ \\
\hline Sodium Hypochlorite & $7681-52-9$ & 1 \\
\hline Sodium Zinc Polyphosphate & $65997-17-3$ & 1,2 \\
\hline Sulfuric Acid & $7664-93-9$ & $1,2,5$ \\
\hline & & \\
\hline Uranium and Uranium Compounds & $7440-61-1$ & $1,2,3$ \\
\hline Urea & $57-13-6$ & 2,3 \\
\hline
\end{tabular}

\section{${ }^{\text {a} H a z a r d ~ C o d e s: ~}$}

1. - Immediate (acute) Health Hazard

2. - Delayed (chronic) Health Hazard

3. - Fire Hazard

4. - Sudden Release of Pressure (compressed gases)

5. - Reactive Hazard

${ }^{b}$ Included also on the basis of its classifications as an asphyxiant. 


\section{Distribution List}

T. A. Bauckham, U.S. West

J. D. Boling

W. L. Clements

T. K. Cothron

S. L. Davis, DOE-ORO

R. A. Evans

R. T. Ford

M. Gallahar, DOE-EOC

P. Gross, DOE-ORO

H. S. Hackler

T. E. Hart

N. K. Kramer, MK-Ferguson

K. J. Martin

T. McWilliams, Johnson Controls

S. M. Peterson

J. D. Rothrock/B. E. Cochran, DOE-ORO

R. L. Sampsel

L. M. Sparks, DOE-ORO

M. Vestal, TDEC

Y-12 Records Services (1)/DOE-OSTI (2)

Y-12 Plant Shift Superintendent

EC Document Control Center - RC 


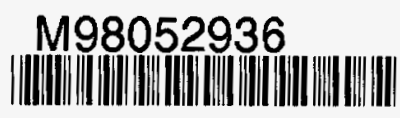

Report Number (14) $1 / 15 \ldots-11058$

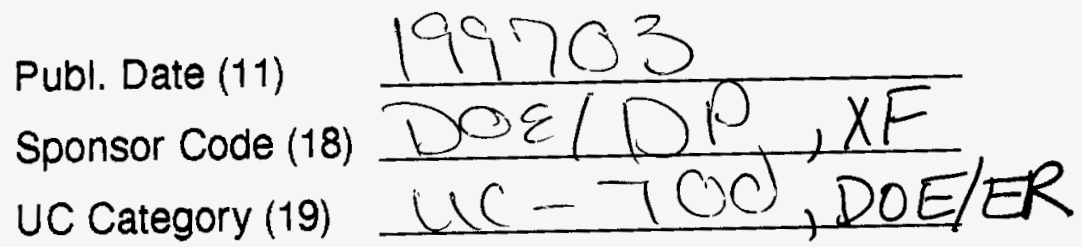

